\title{
Consumo de frutos e abundância de Tucano Toco (Ramphastos toco) em dois hábitats do Pantanal Sul
}

\author{
Leonardo Fernandes França ${ }^{1,4}$, Jose Ragusa-Netto ${ }^{2}$ \& Luciana Vieira de Paiva ${ }^{3}$ \\ ${ }^{1}$ Departamento de Ecologia, Universidade de Brasília - UnB, \\ CEP 70910-900, Brasília, DF, Brasil \\ ${ }^{2}$ Campus Três Lagoas, Universidade Federal de Mato Grosso do Sul-UFMS, \\ Três Lagoas, MS, Brasil, www.ufms.br \\ ${ }^{3}$ Pós-graduação em Ecologia, Instituto de Biologia - IB, Universidade de Brasília - UnB, \\ Brasília, DF, Brasil, www.unb.br \\ ${ }^{4}$ Autor para correspondência: Leonardo Fernandes França, \\ e-mail: franca_lf@yahoo.com,www.unb.br
}

FRANÇA, L.F., RAGUSA-NETTO, J. \& PAIVA, L.V. Toco Toucan (Ramphastos toco) frugivory and abundance in two habitats at South Pantanal. Biota Neotrop., 9(2): http://www.biotaneotropica.org.br/v9n2/ en/abstract?article+bn02109022009.

\begin{abstract}
Toco Toucan (Ramphastos toco) is one of the largest frugivorous birds of canopy, even in continuous forests than in semi-opened habitat. At South Pantanal (Brazil) Toco toucan is common in landscapes that intersperse open and closed habitats. On this research we evaluated the Toco Toucan abundance in capões forest and gallery forest as well as the production and consumption of eshy fruit during dry season. Only six species produced fruits in capões forests, and five in gallery forests. In capões forest the higher presence of Ficus genera, which fructifying was asynchronous, provided constant fruit source. In gallery forests where fig trees were rare, the abundance of eshy fruits was variable. The toucans explored Cecropia pachystachya, Genipa americana and Ficus fruits at two habitats. The occurrence of Toco Toucan in capões forest always was higher than in gallery forest. The occurrence in each habitat was higher when at least two species produced eshy fruits at the same time. The regular toucan occurrence at capões forest was potentially a consequence of constant eshy fruit availability, which occurred due to the presence of Ficus species in this habitat. However, the presence of Toco Toucan at this habitat must result from the combination of all common fruits in bird feed.
\end{abstract}

Keywords: fleshy fruit, forest, frugivorous, frugivory, phenology.

FRANÇA, L.F., RAGUSA-NETTO, J. \& PAIVA, L.V. Consumo de frutos e abundância de Tucano Toco (Ramphastos toco) em dois hábitats do Pantanal Sul. Biota Neotrop., 9(2): http://www.biotaneotropica.org. br/v9n2/pt/abstract?article+bn02109022009.

Resumo: Tucano Toco (Ramphastos toco) é um dos grandes frugívoros de dossel, tanto de orestas contínuas quanto ambientes semi-abertos. No Pantanal Sul o Tucano Toco é comum em paisagens que intercalam ambientes fechados e abertos. Neste estudo avaliamos a abundância de Tucano Toco em capões de mata e mata ciliar, bem como a produção e consumo de frutos carnosos durante a estação seca. Apenas seis espécies frutificaram em capões de mata, e cinco em mata ciliar. Nos capões de mata a maior abundância do gênero Ficus, cuja frutificação foi assincrônica, proporcionou disponibilidade constante de frutos. Na mata ciliar a oferta de frutos carnosos foi variável e as figueiras foram raras e não foram detectadas pelo método de quadrante centrado. Nos dois hábitats os tucanos exploraram frutos de Cecropia pachystachya, Genipa americana e Ficus. A ocorrência de Tucano Toco foi sempre maior em capões de mata do que em mata ciliar, sendo mais elevada em um dado hábitat, quando pelo menos duas espécies disponibilizavam frutos carnosos. A maior regularidade na ocorrência dos tucanos em capões de mata pode ter sido conseqüência da oferta constante de frutos carnosos, a qual ocorreu devido à presença das espécies do gênero Ficus neste hábitat. Porém, a presença da espécie nos hábitats avaliados deve decorrer da combinação de todos os frutos comuns na sua alimentação.

Palavras-chave: fenologia, frugivoria, frugívoro, fruto carnoso, mata. 


\section{Introdução}

A presença e distribuição de frugívoros no ambiente pode estar relacionada aos padrões de abundância dos recursos alimentares (Wheelwright 1983, Levey 1988, Kinnaird et al. 1996, Solórzano et al. 2000). Muitos frugívoros usam frutos extensivamente durante a maior parte do ano, mas a proporção desse recurso na dieta tende a variar durante períodos de escassez, de tal forma que outros itens vegetais ou de origem animal tornam-se mais freqüentes (Terborgh 1986, Galetti et al. 2000, Solórzano et al. 2000). Entre as aves, a movimentação entre manchas de recursos deve ser uma estratégia mais comum que a mudança de hábito alimentar (e.g. Chesser \& Levey 1998). Desta forma, os padrões de distribuição e abundância das espécies de aves frugívoras podem ser explicados tanto pela oferta de frutos nas partes de um ambiente em mosaico como pelo padrão sazonal de disponibilidade de frutos (Wheelwright et al. 1984, Loiselle \& Blake 1990, Kinnaird et al. 1996, Solórzano et al. 2000).

Estudos envolvendo padrões de abundância de aves frugívoras nos Neotrópicos têm enfatizado espécies de pequeno porte do subbosque, estas aves tendem a responder fortemente a oferta de frutos carnosos (e.g. Levey 1988, Loiselle \& Blake 1993). No entanto, as aves frugívoras de médio e grande porte que ocupam o dossel, ambiente cuja frutificação é marcadamente sazonal (Frankie et al. 1974), continuam pouco estudas (porém ver Galetti et al. 2000, Ragusa-Netto 2006). Tucanos (Ramphastidae) são aves de grande porte que ocupam o dossel de matas e cuja dieta está substancialmente baseada em frutos carnosos (Stiles 1993, Galetti et al. 2000, Ragusa-Netto 2006). Estas aves se movimentam por longas distâncias dentro de sua área de vida (Terborgh et al. 1990) visitando árvores em frutificação. Provavelmente, a abundância local de espécies desse gênero é in uenciada pela oferta de frutos (Graham 2001, Galetti et al. 2000, Ragusa-Netto 2006).

O Tucano Toco (Ramphastos toco) é a maior espécie do gênero e ocorre no Cerrado e orestas do interior do Brasil. Diferente de outras espécies que ocorrem em orestas fechadas, o Tucano Toco habita desde áreas semi-abertas até áreas fechadas. Esta espécie é comum no Pantanal (Sick 1997), onde a vegetação está organizada em manchas de orestas dispostas em matrizes de hábitats abertos e semi-abertos (Pott \& Pott 1994). No Pantanal Sul, os capões de mata e a mata ciliar são elementos conspícuos da paisagem. A mata ciliar e capões de mata são estacionais quanto à produção de frutos, porém a ocorrência dos frugívoros locais pode ser afetada pela diferença entre hábitats quanto à diversidade de espécies que produzem frutos carnosos (pers. com.). Poucos estudos enfatizam relações entre oferta de recursos e abundância local de aves frugívoras no Pantanal. Nesse estudo avaliamos, durante a estação seca no Pantanal Sul, a abundância de Tucano Toco em capões de mata e mata ciliar, bem como, a produção e consumo de frutos carnosos nestes ambientes.

\section{Materiais e Métodos}

\section{1. Área de estudo}

O estudo ocorreu na planície inundável do Pantanal Sul em uma área de capões de mata e outra de mata ciliar do Rio Miranda (Município de Corumbá, Estado de Mato Grosso do Sul, Brasil, ca. $19^{\circ} 35^{\prime} \mathrm{S}$ e $57^{\circ} 20^{\prime} \mathrm{W}$, altitude $\pm 100 \mathrm{~m}$ ). Os Capões são áreas cobertas por mata circular de diâmetro em torno de $300 \mathrm{~m}$ ou mata alongada de comprimento inferior a $1.000 \mathrm{~m}$. Estas manchas de mata apresentam dossel variando entre 10 e $15 \mathrm{~m}$ de altura e estão dispostas em uma matriz de áreas abertas composta por gramíneas. As porções de mata ciliar são cordões descontínuos de mata com largura variando entre 50 e 200 m. Estas matas apresentam dossel com altura entre 8 e 13 m e estão intercaladas por áreas abertas cobertas por gramíneas. Delimitamos como área de estudo 15 capões (diâmetros entre 50 a 100 m), e três trechos de mata ciliar (cerca de $1,5 \mathrm{~km}$ de comprimento cada). Os dois ambientes localizavam-se a $20 \mathrm{~km}$ de distância um do outro. Cada capão de mata, assim como cada trecho de mata ciliar, encontrava-se espaçado no mínimo $700 \mathrm{~m}$ um do outro. Os capões de mata tendem a exibir pronunciada deciduidade, principalmente em sua porção central que é mais elevada (Pott \& Pott 1994), enquanto as matas ciliares são semidecíduas, potencialmente devido a menor profundidade do lençol freático (Ragusa-Netto \& Fecchio 2006).

No Pantanal Sul a precipitação média anual é cerca de $1.000 \mathrm{~mm}$ com as chuvas concentradas entre os meses de novembro a março (estação chuvosa) e escassas entre abril a outubro (estação seca). A temperatura média é de $27^{\circ} \mathrm{C}$ na estação chuvosa e de $20^{\circ} \mathrm{C}$ na seca. Nesta região o regime de cheias é anual, se estendendo entre os meses de janeiro e março (Fonte: Divisão de Meteorologia Aeronáutica, Corumbá, MS). As mesmas condições climáticas ocorreram durante o ano do estudo (Figura 1). Todos os dados do estudo foram coletados entre os meses de abril e novembro de 2002, nos nove primeiros dias de cada mês. Portanto, o estudo esteve compreendido entre o início da estação seca e a transição para a estação úmida.

\section{Disponibilidade de frutos carnosos}

Para avaliar a oferta de frutos selecionamos todas as espécies arbóreas que produzem frutos carnosos durante a estação seca nas áreas de estudos (Pott \& Pott 1994, Ragusa-Netto \& Fecchio 2006). Isto porque não conhecíamos a relevância de cada planta para a alimentação de Tucano Toco. Na perspectiva de amostrar árvores do dossel individualizamos com plaquetas de alumínio numeradas as árvores com diâmetro acima do peito (DAP) superior a $20 \mathrm{~cm}$ e altura superior a 3 metros. Amostramos pelo menos cinco indivíduos de cada espécie, por constituir uma amostra minimamente representativa. No entanto, para algumas espécies abundantes ou de frutificação assincrônica selecionamos até 20 árvores. Por outro lado, para algumas espécies pouco comuns marcamos todos os indivíduos encontrados mesmo que não atingissem o número mínimo previamente estabelecido. Em capões de mata, marcamos indivíduos de Cecropia pachystachya $(\mathrm{N}=13)$, Ficus. calyptrocera (4), F. luschnathiana (20), F. pertusa (23), Inga vera (7) e Sterculia apetala (10). Em mata ciliar marcamos indivíduos

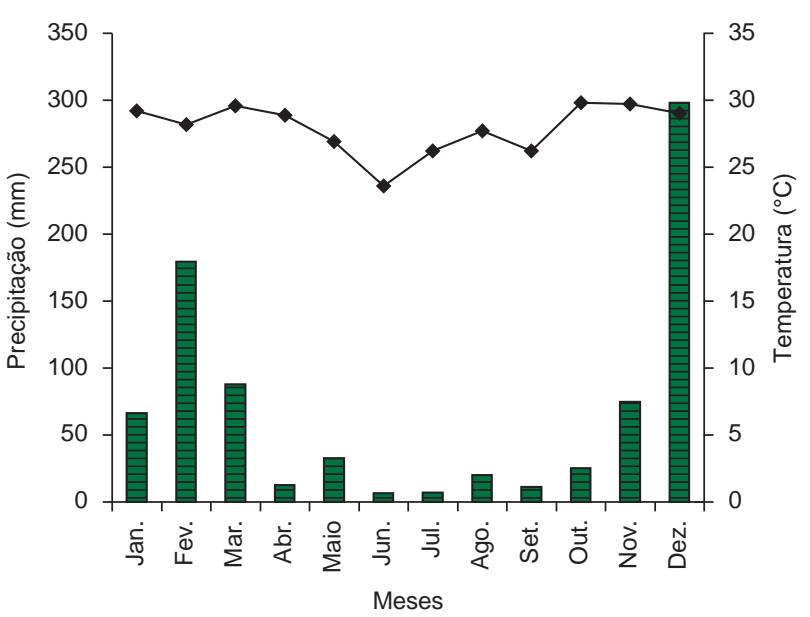

Figura 1. Precipitação mensal (barra) e temperatura média (linha) no Pantanal Sul em 2002. Fonte: Divisão de meteorologia aeronáutica, Brasil.

Figure 1. Mensal precipitation (bar) and average temperature (line) at South Pantatnal in 2002. Source: Divisão de meteorologia aeronáutica, Brazil. 
de C. pachystachya (16), Genipa americana (13), Copernicia alba (17), I. vera (13) e F. luschnathiana (2). Genipa americana não foi marcada nos capões, pois os indivíduos encontrados inicialmente não estavam frutificando. Porém, em capões não amostrados, os indivíduos frutificaram e os frutos amadureceram de forma semelhante à observada em mata ciliar (pers. com.). Dessa forma, nos capões, essa espécie foi amostrada apenas quanto ao consumo de frutos pelos tucanos. Apenas para efeito de comparação, assumimos que a produção de frutos de G. americana foi similar em ambos hábitats, pois a densidade de indivíduos diferiu pouco entre ambientes (ver Tabela 1). As espécies do gênero Ficus foram avaliadas conjuntamente quanto à produção de frutos e incluímos um número superior de indivíduos para obter uma amostra representativa do processo assincrônico de frutificação. Também amostramos um maior número de indivíduos de C. pachystachya, pois tende a frutificar pelo menos 2 vezes durante a estação seca (Ragusa-Netto 2006).

Cada árvore foi monitorada mensalmente (entre os cinco primeiros dias, de abril a novembro de 2002) quanto à presença de frutos maduros. Para tal, atribuímos escores de abundância de frutos os quais variaram de zero a quatro, sendo zero a ausência de frutos, um correspondente de 01 a $25 \%$ da copa ocupada com frutos, dois correspondente de 26 a $50 \%$ e assim progressivamente (Fournier 1974). Com isso, padronizamos os dados e obtivemos o índice mensal de abundância, a partir da soma dos escores dividido pelo número de indivíduos amostrados mensalmente em cada espécie.

\section{Abundância de espécies com frutos carnosos}

Utilizamos o método do quadrante centrado para avaliar a importância das espécies arbóreas nas duas fisionomias vegetais. Em cada ambiente coletamos dados em 20 pontos. Em capões de mata os pontos foram estabelecidos próximo à borda e no centro e, na mata ciliar, foram estabelecidos a uma distância de $15 \mathrm{~m}$ da margem. Ao todo foram avaliados 10 capões de mata e dois trechos de mata ciliar dentre aqueles do grupo previamente estabelecido. Em cada quadrante mediamos a distância do ponto central até a árvore mais próxima e a circunferência à altura do peito (CAP) desta árvore. Identificamos as espécies e agrupamos aquelas que não faziam parte do estudo em uma categoria denominada outros. Utilizando estes dados calculamos o Índice de Valor de Importância (IVI) para determinar a importância de cada espécie estudada em cada ambiente. Este índice é adequado para caracterização de fisionomias arbóreas.

\section{Abundância de tucanos}

Avaliamos a abundância mensal de tucanos através da técnica de censo por ponto. $\mathrm{O}$ censo por ponto é normalmente recomendado

Tabela 1. Índice de Valor de Importância para espécies arbóreas em capões de mata e mata ciliar do Pantanal Sul, (N = 20 quadrantes em cada hábitat).

Table 1. Index Value of Importance to tree species in Capões Forest and Gallery Forest at South Pantanal, ( $\mathrm{N}=20$ quadrants in each habitat).

\begin{tabular}{lcc}
\hline \multicolumn{1}{c}{ Espécies } & \multicolumn{2}{c}{$\begin{array}{c}\text { Índice de Valor de Importância } \\
\text { (IVI) }\end{array}$} \\
\cline { 2 - 3 } & Capões de mata & Mata ciliar \\
\hline Copernicia alba & 0,00 & 7,26 \\
Cecropia pachystachya & 15,80 & 41,88 \\
Ficus spp & 13,82 & 0,00 \\
Ingá Vera & 10,33 & 93,59 \\
Genipa americana & 5,02 & 6,67 \\
Sterculia apetala & 9,62 & 0,00 \\
Outras & 245,40 & 150,60 \\
\hline
\end{tabular}

para determinar a abundância de grandes aves frugívoras de dossel em ambientes fechados (Marsden 1999). Estabelecemos 15 pontos em áreas de capões de mata e 15 em áreas de mata Ciliar. Os pontos foram delimitados nas mesmas rotas usadas para o estudo fenológico e distavam entre si pelo menos $300 \mathrm{~m}$ dentro de cada hábitat. Mensalmente as amostragens ocorreram entre 06:30 e 08:30 horas e em cada ponto contávamos durante 10 minutos todos os tucanos avistados ou ouvidos a uma distância máxima de $300 \mathrm{~m}$. Mensalmente amostramos cada ponto uma única vez e determinamos a seqüência de amostragem por sorteio sem reposição. Definimos como índice mensal de abundância de tucanos em cada hábitat, a razão entre o número total de tucanos detectados e o número de pontos amostrados.

\section{Consumo de frutos}

Amostramos o uso de recurso alimentar por Tucano Toco tanto nos capões de mata quanto na mata Ciliar. Para isto fizemos observações diretas de consumo ao longo de trilhas estabelecidas nos mesmos trechos de mata já mencionados. Estas observações foram feitas com o auxílio de binóculos $8 \times 40$ e ocorreram de forma independente dos censos por ponto. Durante a amostragem sempre que um tucano era detectado passava a ser seguido na perspectiva de que se alimentasse. Consideramos como um evento alimentar o consumo de um determinado item independentemente da quantidade de alimento ingerido ou do tempo que o indivíduo permanecia consumindo o recurso. Durante as observações registrávamos: 1) número de indivíduos avistados, 2) número de indivíduos se alimentando, 3) espécie da planta e 4) item consumido ( or, arilo e polpa). Utilizamos apenas o primeiro registro de alimentação de cada indivíduo para obter amostras independentes de consumo do recurso.

\section{Resultados}

\section{Disponibilidade de frutos carnosos}

$\mathrm{Na}$ área com capões de mata, C. pachystachya e as espécies do gênero Ficus frutificaram durante toda estação seca, porém a frutificação em $C$. pachystachya declinou durante os meses mais secos (junho a setembro, Figura 2a). Em média 10 de um total de 49 árvores do gênero Ficus frutificaram a cada mês, caracterizando um padrão assincrônico dentro do gênero. Inga vera frutificou moderadamente em dois meses nesse período e os indivíduos de $S$. apetala marcados, não produziram frutos maduros. Apesar dos indivíduos de G. americana amostrados nos capões não terem frutificado, outros indivíduos produziram frutos nessa área de forma semelhante ao que ocorreu na mata ciliar.

Na mata ciliar $C$. pachystachya produziu frutos durante toda estação seca, porém a oferta de frutos declinou de meados para o final dessa estação (Figura 2b). Em C. alba os frutos amadureceram de abril a junho, enquanto que em $G$. americana a disponibilidade de frutos maduros foi maior em meados da estação seca. Os indivíduos de I. vera frutificaram esporadicamente no início e meio da estação seca. Os dois indivíduos de $F$. luschnatiana encontrados frutificaram apenas em setembro.

\section{Abundância de espécies com frutos carnosos}

Nos capões de mata, C. pachystachya e o gênero Ficus foram as espécies vegetais mais importantes (Tabela 1) dentre as consumidas por Tucano Toco (Figura 3). O IVI agrupado das espécies que frutificaram neste ambiente correspondeu cerca de $19 \%$ do total de indivíduos presentes na área. Das espécies consumidas em mata ciliar (Figura 3), C. pachystachya foi pelo menos 6 vezes mais abundante que $G$. americana, a segunda espécie mais importante segundo o índice utilizado (Tabela 1). O IVI agrupado das espécies 

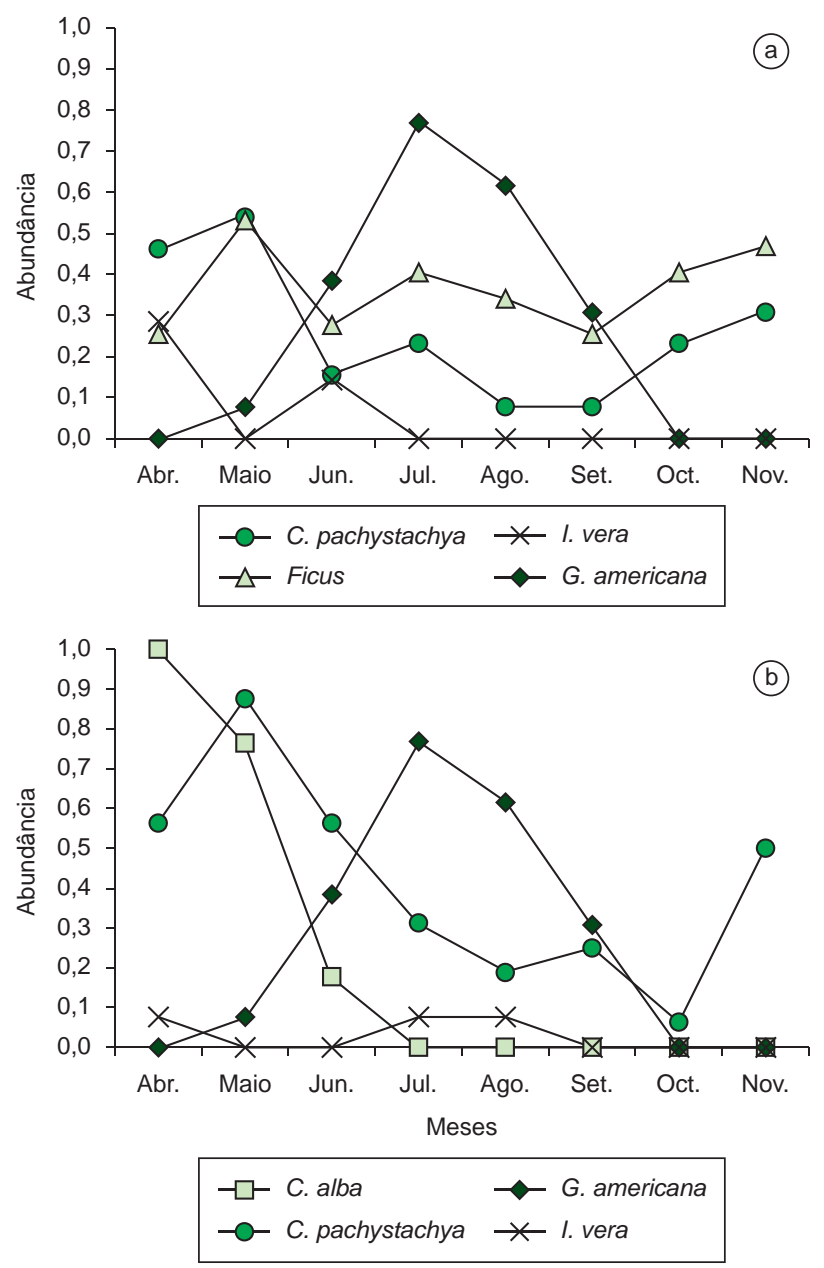

Figura 2. Abundância de frutos maduros consumidos por Tucano Toco durante a estação seca (de abril a novembro de 2002) em a) capões de mata e b) mata ciliar do Pantanal Sul.

Figure 2. Ripe fruit abundance consumed by Toco Toucan along dry season (from April to November 2002) in Capões Forest (above) and Gallery Forest (below) at South Pantanal.

que frutificaram neste ambiente correspondeu cerca de $11 \%$ do total de indivíduos registrados.

\section{Consumo de frutos}

Ao todo amostramos consumo de fruto durante 97:35 horas em capões de mata e 72:37 horas em mata ciliar. Nos capões avistamos o Tucano Toco 153 vezes, das quais consumiram frutos 61 vezes $(0,62$ registros/ hora de observação). Na mata ciliar registramos a ave 56 vezes, das quais 16 consumindo frutos (0,22 registros/ hora de observação). Com exceção do registro de consumo de uma única cigarra (Hemiptera), os tucanos sempre consumiram frutos. Nos dois ambientes os frutos maduros de C. pachystachya, G. americana e Ficus foram consumidos nesta respectiva ordem de importância, sendo os frutos da primeira espécie pelo menos 4 vezes mais consumidos (Figura 3). Em capões de mata registramos um único consumo de frutos de $S$. apetala.

Tucanos consumiram frutos em capões de mata e mata ciliar com maior freqüência em meados da estação seca. Em capões de mata $81 \%$ dos registros de consumo ocorreram de julho a setembro, quando frutificavam simultaneamente pelo menos duas espécies dentre as mais consumidas por tucanos. Em julho, tucanos consumiram C. pachystachya e G. americana $(\mathrm{N}=23$ indivíduos consumindo), em

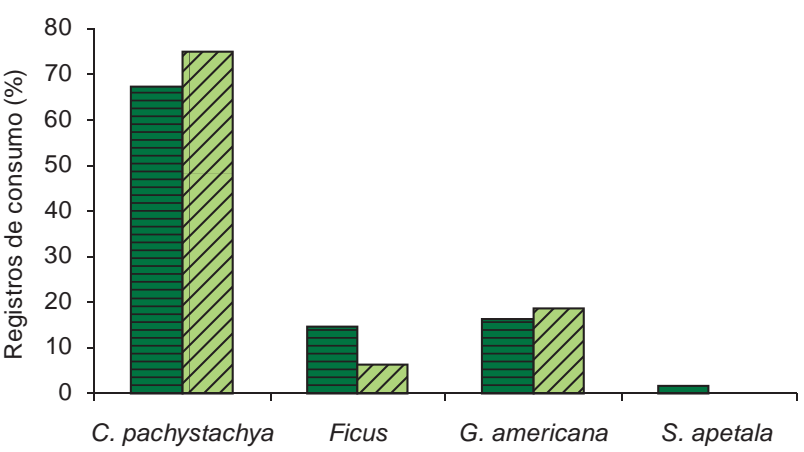

Figura 3. Porcentagens de frutos consumidos por Tucano Toco durante a estação seca (de abril a novembro de 2002) em capões de mata (barras verde escuro, $\mathrm{N}=61,97: 35 \mathrm{~h} /$ homem de observação) e mata ciliar (barras verde claro, $\mathrm{N}=16,72: 37$ horas/ homem) do Pantanal Sul.

Figure 3. Percentage of fruit eaten by Toco toucan on dry season (from April to November 2002) in Capões Forest (dark green bar, $\mathrm{N}=61,97: 35$ hours/ observation) and Gallery Forest (light green bar, $\mathrm{N}=16,72: 37 \mathrm{~h} /$ observation) at South Pantanal.

agosto C. pachystachya, G. americana e Ficus spp $(\mathrm{N}=14)$ e em setembro consumiram C. pachystachya, Ficus spp e S. apetala $(\mathrm{N}=12)$. Na mata ciliar $70 \%$ dos registros de consumo ocorreram durante os meses de junho, julho e setembro, período em que C. pachystachya e G. americana disponibilizavam frutos maduros.

\section{Abundância de Tucano Toco}

A abundância mensal de tucanos em capões de mata foi sempre maior que a abundância em mata ciliar (Figura 4). A espécie esteve presente durante toda a estação seca nos capões de mata, inclusive no período de menor riqueza de frutos (outubro e novembro) e ausência da ave em mata ciliar (Figura 2b, Figura 4). Nos capões a abundância de Tucano Toco nunca foi igual a zero e a oscilação entre meses nunca excedeu 0,5 indivíduos por ponto (Figura 4). Neste hábitat, a produtividade de frutos carnosos resultou na disponibilidade mensal de pelo menos três tipos de frutos em seis dos oito meses de estudo (Figura 2a). Em todos os meses avaliados estiveram disponíveis pelo menos dois tipos de frutos dentre os consumidos por Tucano Toco (Figura 2a).

Tucano Toco ocorreu na mata ciliar de abril a agosto (Figura 4), quando pelo menos duas espécies frutificavam em abundância (Figura 2b). O mês em que Tucano Toco foi mais abundante na mata ciliar (junho) coincidiu com a elevada abundância dos frutos de C. alba e dos frutos mais consumidos pela ave (C. pachystachya $\mathrm{e}$ G. americana). De abril a agosto, exceto junho, a abundância de tucanos ficou entre 0,07 e 0,13 indivíduos por ponto (Figura 4). Os dois primeiros meses corresponderam ao período de maior abundância de frutos de $C$. pachystachya e produção de frutos maduros de C. alba, enquanto os dois últimos meses (julho e agosto) corresponderam a períodos de alta abundância de frutos maduros de C. pachystachya e G. americana. Tucano Toco se tornou ausente da mata ciliar no período em que a frutificação em $G$. americana e $C$. pachystachya declinou abruptamente (setembro e outubro) e quando C. pachystachya era a única espécie a disponibilizar frutos maduros (novembro, Figura $2 b$ ).

\section{Discussão}

\section{Disponibilidade de frutos carnosos}

Em ambientes tropicais úmidos a produção de frutos carnosos é geralmente menor durante a estação seca (Blake \& Loiselle 1991, 


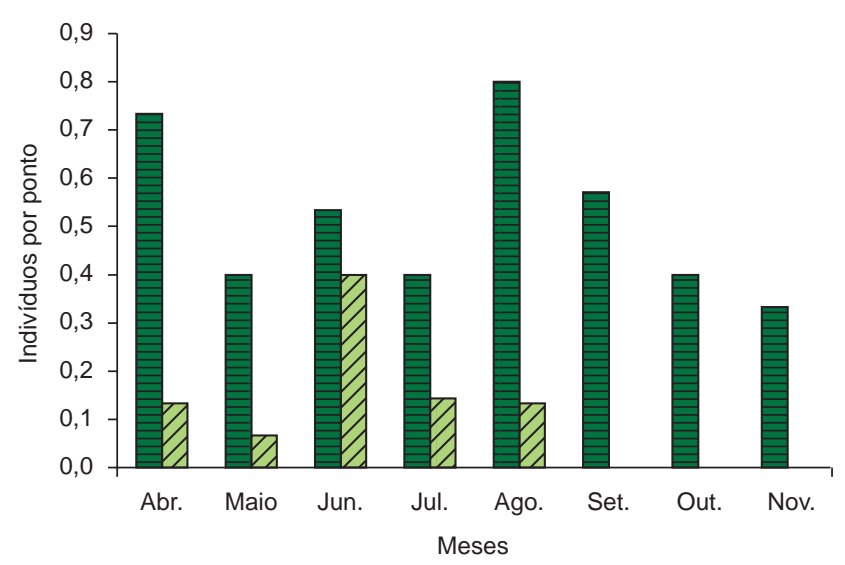

Figura 4. Abundância mensal (de abril a novembro de 2002) de Tucano Toco durante a estação seca em capões de mata (barras verde escuro) e mata ciliar (barras verde claro) do Pantanal Sul. $\mathrm{N}=15$ pontos por habitat.

Figure 4. Mensal abundance (from April to November 2002) of Toco toucan on dry season in Capões Forest (dark green bar) and Gallery Forest (light green bar) at South Pantanal. $\mathrm{N}=15$ points per habitat.

Garber 1993, Peres 1994) e o mesmo ocorre em áreas com precipitação marcadamente sazonal (Funch \& Funch 2002). As matas avaliadas neste estudo apresentaram vegetação semelhante à observada em ambientes tropicais secos (Pott \& Pott 1994). Os trechos de mata ciliar exibiram um padrão de frutificação típico de ambientes sazonais, por outro lado, os capões de mata apesar de altamente decíduos, disponibilizaram frutos durante todo período seco. A maior constância temporal de frutos nos capões de mata se deveu a grande quantidade de figueiras, as quais frutificaram durante o período de declínio na frutificação nas demais espécies.

A disponibilidade mensal de frutos em cada ambiente resultou da combinação da oferta de frutos estacionais e assincrônicos. Dessa forma, pelo menos frutos maduros de C. pachystachya e Ficus spp. foram produzidos mesmo nos meses mais rigorosos. Isto ocorreu devido ao padrão de frutificação dessas espécies, ou seja, ciclo múltiplo em C. pachystachya (Ragusa-Netto 2006, Carlo et al. 2004), e frutificação assincrônica em Ficus spp (Milton et al. 1982, Kinnaird et al. 1996, Ragusa-Netto 2002). As demais espécies incrementaram os níveis de oferta em alguns meses, sendo que combinadas com C. pachystachya, na mata ciliar, ou Ficus, nos capões, configuraram uma importante fonte de alimento em ambos os hábitats. A elevada densidade de Ficus nos Capões caracterizou-se como um diferencial que aponta este hábitat como particularmente importante para o Tucano Toco durante a estação seca.

O conjunto de espécies que potencialmente frutifica durante a seca é pequeno quando comparado ao que usualmente frutifica em orestas tropicais úmidas (e.g. Levey 1988, Peres 1994) ou ambientes tropicais secos (Justiniano \& Frederichsen 2000, Funch \& Funch 2002). Por outro lado, os indivíduos destas espécies estiveram entre os mais abundantes, tanto nos capões quanto na mata ciliar. Além disso, são indivíduos que tendem a investir massivamente em diásporos (Martin 1985, Levey 1988), por serem de espécies pioneiras ou de vegetação secundária (Pott \& Pott 1994). Estas características conferiram aos ambientes uma baixa riqueza, porém uma abundante oferta de frutos carnosos. Caracterizando assim os hábitats avaliados, como importantes áreas de alimentação para o Tucano Toco durante a estação seca.

\section{Frutos carnosos e abundância de tucanos}

Nos dois hábitats os tucanos foram avistados explorando predominantemente frutos carnosos, a exemplo do que ocorre com outras espécies do gênero em orestas úmidas (Ramphastos vitellinus e $R$. dicolorus; Galetti et al. 2000). Os tucanos praticamente não foram vistos predando artrópodes ou pequenos vertebrados, apesar desse estudo ter sido desenvolvido durante a estação seca quando a propensão ao consumo de itens de origem animal aumenta. Os itens de origem animal foram mais comuns na dieta de $R$. sulphuratus em matas secas (Skutch 1971). A manutenção de uma dieta frugívora em lugar de mudança nos hábitos alimentares é consistente com o uso de um mosaico de hábitats no qual ocorre uma oferta adequada de frutos (Van Schaik et al. 1993). Em diversas ocasiões observamos tucanos cruzando áreas abertas em direção à mata ciliar ou em direção aos capões de mata. Embora esses indivíduos não estivessem marcados é plausível que tais movimentos re itam o percurso de rotas de alimentação (Graham 2001). As rotas de alimentação são típicas de espécies que usam grandes áreas de vida com manchas de recursos alimentares (Terborgh et al. 1990, Van Schaik et al. 1993), tal como ocorre em Tucano Toco.

Durante a estação seca nas orestas tropicais úmidas, a riqueza de espécies que disponibilizam frutos carnosos para os ranfastídeos é normalmente maior (Galetti et al. 2000) do que a encontrada para o Tucano Toco no Pantanal Sul. Portanto, a dieta de Tucano Toco pode ser considerada pobre em número de tipos de frutos consumidos, visto que esteve restrita principalmente a quatro (Cecropia pachystachya, Genipa americana e dois tipos de Ficus spp) ou até no máximo seis tipos de frutos (incluindo S. apetala e C. alba). Possivelmente, também seja pobre na sua composição nutricional visto que os frutos consumidos são de espécies com padrão generalista de dispersão. Neste padrão estão incluídos caracteristicamente frutos de baixo teor nutricional e energético (Howe 1993). Por outro lado, a alta produção de diásporos compensa o baixo teor de lipídeos e proteínas de frutos com baixo teor nutricional (Stiles 1993), o que deve suprir as necessidades de fruto na dieta do Tucano Toco e garantir sua ocorrência na área estudada durante o período seco.

A abundância de figueiras nos capões e respectiva escassez na mata ciliar pode ter sido o fator mais importante para a elevada ocorrência dos tucanos nas áreas de capões de mata. Os hábitats em que figueiras são abundantes e a oferta de figos é permanente, normalmente abrigam densidades mais elevadas de aves frugívoras de dossel (Kinnaird et al. 1996). A importância dos frutos de figueiras para ranfastídeos é sugerida por alguns estudos em que esse recurso foi amplamente consumido (Coates-Estrada \& Estrada 1986, Bronstein \& Hoffmann 1987) e o mesmo parece válido para o Tucano Toco (Ragusa-Netto 2002). Apesar da qualidade nutricional dos figos ser baixa, algumas espécies exibem alto teor de lipídeos e proteínas. A combinação de alguns tipos de figos pode proporcionar a qualidade nutricional adequada para suprir os requisitos alimentares de espécies altamente frugívoras (Wendeln \& Runkle 2000). No Pantanal, os frutos de figueiras freqüentemente combinados com os de $C$. pachystachya e $G$. americana podem conferir uma dieta consistente que, pelo menos em parte, explique a persistência de Tucano Toco no mosaico de hábitats durante o período de baixa disponibilidade de outros frutos.

\section{Agradecimentos}

Aos funcionários da Base de Estudos do Pantanal: Edílson, Dona Josefa, Rosana e Rosimar, pelos serviços prestados. Aos proprietários da Fazenda São João e Passo do Lontra: Jose Venturine, João Buzinhani e Alison Buzinhani por permitirem pleno acesso aos locais 
e fornecerem apoio logístico. À Andréia Araújo e Celine Melo pelas revisões do texto e à Capes pela bolsa concedida.

\section{Referências Bibliográficas}

BLAKE, J.G. \& LOISELLE, B.A. 1991. Variation in resource abundance affects capture rates of birds in three lowland habitats in Costa Rica. Auk. 108(1):114-130.

BRONSTEIN, J.L. \& HOFFMANN, K. 1987. Spatial and temporal variation in frugivory at a neotropical fig, Ficus pertusa. Oikos. 49(3):261-268.

CARLO, T.A., COLLAZO, J.A. \& GROOM, M.J. 2004. In uences of Fruit Diversity and Abundance on Bird Use of Two Shaded Coffee Plantations. Biotropica. 36(4):602-614.

CHESSER, R.T. \& LEVEY, D.J. 1998. Austral migrants and the evolution of migration in new world birds: diet, habitat, and migration revisited. Am. Nat. 152(2):311-319.

COATES-ESTRADA, R. \& ESTRADA, A. 1986. Fruiting and frugivores at a strangler fig in the tropical rain forest of Los Tuxtlas, Mexico. J. Trop. Ecol. 2(4):349-357.

FRANKIE, T.H., BAKER, H.G. \& OPLER, P.A. 1974. Comparative phenological studies of trees in tropical wet and dry forest in the lowlands of Costa Rica. J. Ecol. 62(3):881-919.

FOURNIER, L.A. 1974. Un metodo cuantitativo para la medición de características fenologicas em arboles. Turrialba (II CA). 24:422-423.

FUNCH, L.S. \& FUNCH, R. 2002. Phenology of gallery and montane forest in the Chapada Diamantina, Bahia, Brazil. Biotropica. 34(1):40-50.

GALETTI, M., LAPS, R. \& PIZO, M.A. 2000. Frugivory by toucans (Ramphastidae) at two altitudes in the Atlantic forest of Brazil. Biotropica. 32(4):842-850.

GARBER, P.A. 1993. Seasonal patterns of diet and ranging in two species of Tamarin monkeys: stability versus variability. Int. J. Primatol. 14(1):145-166.

GRAHAM, C.H. 2001. Factor in uencing movement patterns of Kell-billed Toucans in fragmented tropical landscape in southern Mexico. Conserv. Biol. 15(6):1789-1798.

HOWE, H.F. 1993. Specialized and generalized dispersal systems: where does 'the paradigm' stand? In Frugivory and seed dispersal: Ecological and Evolutionary aspects (T.H. Fleming \& A. Estrada, eds). Kluwer Academic Publishers, Belgium, p. 3-13.

JUSTINIANO, M.J. \& FREDERICKSEN, T.S. 2000. Phenology of tree species in Bolivian dry forests. Biotropica. 32(2):276-281.

KINNAIRD, M.F., O'BRIEN T.G. \& SURYADI, S. 1996. Population uctuation in Sulawesi Red-knobbed Hornbills: tracking figs in space and time. Auk. 113(2):431-440.

LEVEY, D.J. 1988. Spatial and temporal variation in Costa Rican fruit and fruit-eating birds abundance. Ecol. Monogr. 58(4):251-269.

LOISELLE, B.A. \& BLAKE, J.G. 1993. Spatial distribution of understory fruit-eating bird and fruiting plants in a neotropical lowland wet forest. In Frugivory and seed dispersal: Ecological and Evolutionary aspects (T.H. Fleming \& A. Estrada, eds). Kluwer Academic Publishers, Belgium, p. $177-189$.
LOISELLE, B.A. \& BLAKE, J.G. 1990. Diets of understory fruit-eating birds in Costa Rica: seasonality and resource abundance. Stud. Avian Biol. 13(13):91-103.

MARSDEN, S.J. 1999. Estimation of parrot and hornbill densities using a point count distance sampling method. Ibis. 141(3):377-390.

MARTIN, T.E. 1985. Selection of second-growth woodlands by frugivorous migrating birds in Panama: an effect of fruit size and plant density? J. Trop. Ecol. 1(2):157-170.

MILTON, K., WINDSOR, D.M., MORRISON, D.W. \& ESTRIBI, M.A. 1982. Fruiting phenologies of two Neotropical Ficus species. Ecology. 63(3):752-762.

PERES, C.A. 1994. Primate responses to phenological changes in an Amazonian terra firme forest. Biotropica. 26(1):98-112.

POTT, A. \& POTT, V.J. 1994. Plantas do Pantanal. Centro de pesquisa agropecuária do Pantanal (Embrapa), Corumbá.

RAGUSA-NETTO, J. 2002. Fruiting phenology and consumption by birds in Ficus calyptroceras (Miq.) Miq. (Moraceae). Braz. J. Biol. 62(2):339-346.

RAGUSA-NETTO, J. 2006. Abundance and frugivory of the Toco toucan (Ramphastos toco) in a gallery Forest in Brazil's Pantanal. Braz. J. Biol. 66(1):133-142.

RAGUSA-NETTO, J., FECCHIO, A. 2006. Plant food resources and the diet of a parrot community in a gallery forest of the southern Pantanal (Brazil). Braz. J. Biol. 66(4):1021-1032.

SICK, H. 1997. Ornitologia Brasileira. Nova Fronteira, Rio de Janeiro.

SKUTCH, A.F. 1971. Life history of the Keel-billed Toucan. Auk. 88(2):381-424.

SOlóRzANO, S., CASTILlo, S., VAlVERDE, T. \& ÁVILA, L. 2000. Quetzal abundance in relation of fruit availability in a cloud forest in Southeastern Mexico. Biotropica. 32(3):523-532.

STILES, H.W. 1993. In uence of pulp lipids on fruit preferences by birds. Vegetatio. 107-108(1):227-236.

TERBORGH, J. 1986. Keystone plant resources in the tropical forest. In Conservation biology: the science of scarcity and diversity (M.E. Soulé, ed). Sinauer Associates, Sunderland, p. 33-44.

TERBORGH, J., ROBINSON, S.K., PARKER III, T.A., MUNN, C. \& PIERPOINT, N. 1990. Structure and organization of an Amazonian forest bird community. Ecol. Monogr. 60(2):213-238.

VAN SCHAIK, C.P., TERBORGH, J.W. \& WRIGHT, S.J. 1993. The phenology of tropical forests: adaptive significance and consequences for primary consumers. Annu. Rev. Ecol. Syst. 24:353-377.

WENDELN, M.C. \& RUNKLE, J.R. 2000. Nutritional values of 14 fig species and bat feeding preferences in Panama. Biotropica. 32(3):489-501.

WHEELWRIGHT, N.T. 1983. Fruits and the ecology of Resplendent Quetzals. Auk. 100(2):286-301.

WHEELWRIGHT, N.T., HABER, W.A., MURRAY, K.G. \& GUINDON, C. 1984. Tropical fruit-eating birds and their food plants: A survey of a Costa Rican lower montane forest. Biotropica. 16(3):173-192.

Recebido em 30/10/08 Versão reformulada recebida em 07/04/09 Publicado em: 12/05/09 